\title{
Minimally Invasive Pancreatic Resection: Is It Really the Future?
}

\author{
G. Nappo J.Perinel M. El Bechwaty M. Adham \\ Department of HBP Surgery, Edouard Herriot Hopsital, HCL and Lyon Faculty of Medicine, Lyon, France
}

\section{Key Words}

Pancreatectomy · Laparoscopy · Minimally invasive

\begin{abstract}
The introduction and widespread application of minimally invasive surgery has been one of the most important innovations that radically changed the practice of surgery during the last few decades. The application to pancreatic surgery of minimally invasive approach has only recently emerged: both laparoscopic distal pancreatectomy (LDP) and laparoscopic pancreaticoduodenectomy (LPD) can be competently performed. LDP and LPD are advocated to improved perioperative outcomes, including decreased blood loss, shorter length of stay, reduced postoperative pain and expedited time to functional recovery. However, the indication to minimally invasive approach for pancreatic surgery is often benign or low-grade malignant pathologies. In this review, we summarize the current data on minimally invasive pancreatic surgery, focusing on indication, perioperative and oncological outcomes.

(c) 2016 S. Karger AG, Basel
\end{abstract}

The introduction and widespread application of minimally invasive surgery has been one of the most important innovations that radically changed the practice of surgery during the last few decades. From the first laparoscopic cholecystectomy by Philippe Mouret in 1987 [1], laparoscopy has been widely implemented and has resulted in improvements in perioperative outcomes for a wide variety of operative procedures. Pancreatic resections are considered, up to now, great challenges for the surgeons, due to their complexity and the severity of postoperative complications. More than 2 decades after the beginning of 'laparoscopic era', is minimally invasive approach feasible and safe for pancreatic surgery? To date, the answer is probably 'Yes, it is possible!' in selected cases and in expert hands. However, more difficult is to answer to the following questions: 'Can minimally invasive approach for pancreatic surgery be considered comparable with the open approach? Is this approach oncologically safe in patients with malignancy? Several studies have been published in the last years, suggesting that the benefits of laparoscopy may be true for pancreatic surgery as well [2]. The subsequent adoption of robotic assistance further extended the advantages of laparoscopic approach, by providing the surgeon with increased dexterity and range of motion of articulating instruments and by improving quality image of the surgical field thanks to $3 \mathrm{D}$ visualization [3]. These theoretical advantages of the robot are particularly helpful for minimally invasive pancreatectomies, and for these reasons, the development of robotic surgery improved the trend of pancreatic surgeons to perform major minimally invasive pancreatic resections. However, the use of these mini-invasive techniques in the field of pancreatic surgery has been slow to develop, and up to now, few specialized centers routinely perform a

\section{KARGER}

E-Mail karger@karger.com www.karger.com/dsu
(C) 2016 S. Karger AG, Basel

0253-4886/16/0334-0284\$39.50/0
G. Nappo

HBP Surgery, Edouard Herriot Hospital

5 Place d'arsonval, Pavillon D2

FR-69437 Lyon Cedex 03 (France)

E-Mail g.nappo@unicampus.it 
minimally invasive approach for major pancreatic resections. Some reasons can be adducted. Firstly, the complexity of pancreatic resections and, particularly, of pancreaticoduodenectomy (PD), due to the retroperitoneal location of the pancreas, its proximity to major vessels and the necessity to perform complicated anastomotic components. Secondly, it is clear that minimally invasive approach is safe and feasible, but it is not yet demonstrated that it is 'oncologically effective'; no randomized controlled trials (RCTs) studies are present in the literature, and many retrospective published studies selected about minimally invasive approach consider only benign or borderline lesions. So, long-term outcomes of minimally invasive pancreatic resections for malignancy are lacking. Furthermore, an additional longstanding criticism of the minimally invasive approach, particularly for the robotic one, is elevated costs and the lack of a robust cost-benefit analysis $[4,5]$.

For all these considerations, the debate about the use of minimally invasive approach for pancreatic surgery still remains open. We report the state of the art on the use of minimally invasive approach for PD and distal pancreatectomy (DP), with particular attention not only to feasibility and safety, but also to indications, post-operative outcomes and long-term results of these 2 procedures.

\section{Minimally Invasive DP}

DP is the most common pancreatic procedure performed by minimally invasive approach. This is clearly comprehensible: it is easier to perform, it requires no reconstruction and it is technically less challenging and more readily adopted by pancreatic surgeons with laparoscopic training and skills, if compared with a more complex procedure as PD.

Laparoscopic DP (LDP) was first performed by Cuschieri et al. [6] for benign disease in 1996, and in the same year, Gagner et al. [7] published their early experience with the first $8 \mathrm{LDP}$ for patients with islet cell tumors. In this study, the author well documented the problems of this new approach - high conversion rate $(37 \%)$ and long operating time (mean time $4.5 \mathrm{~h}$ ) - but also a remarkable reduction in terms of length of stay (mean 5 days) was observed. After the publications of these encouraging results, the attention toward LDP has dramatically increased and numerous case series, each comprising more than 100 surgically treated patients, have been published [8-12]. These studies supported that LDP is technically feasible and safe in expert hands. Moreover, with the acquisition of technical skills, the average operative time and the conversion rate are significantly reduced during the last decade. For all these reasons, many centers have adopted the laparoscopic approach as the standard procedure for patients undergoing DP.

It is easier to demonstrate the feasibility and safety of LDP, but more difficult is to verify if this surgical approach determines real advantages in terms of postoperative outcomes and can be considered a true alternative to open approach. In fact, looking to the available literature, the comparisons of outcomes between LDP and open DP (OPD) are currently limited to retrospective studies and no prospective RCTs have been published [11, 1316]. Stauffer et al. [17] performed a comparative study, which included 82 LDP and 90 ODP; LDP was associated with significantly decreased blood loss ( 180 vs. $880 \mathrm{ml}$; $\mathrm{p}<0.001)$ and thus fewer transfusions ( 0.35 vs. 2.5 units; $\mathrm{p}<0.001)$, shorter intensive care unit ( 0.1 vs. 0.7 days; $\mathrm{p}<$ 0.001 ) and shorted hospital stays (4.3 vs. 8.1 days; $\mathrm{p}<$ 0.001 ), while rates of grade $\mathrm{B} / \mathrm{C}$ postoperative pancreatic fistula were similar in both groups (6 vs. $10 \%)$. Similar results were described by Kooby et al. [8] and Jayaraman et al. [11], confirming a significant reduction in blood loss and hospital length of stay in the laparoscopic group.

Although RCTs comparing LDP and ODP are still not available, many meta-analyses of retrospective studies have been published [18-25]. One of them was recently published in 2012 by Venkat et al. [20], which reviewed 1,814 patients from 18 comparative trials. This meta-analysis, confirmed the excellent results of laparoscopic approach: significantly less blood loss, shorter length of hospital stay and lower rate of overall complications (particularly, fewer surgical site infections). Interestingly, specifically looking at pancreatic fistulas, the authors found no difference in terms of incidence of clinically relevant (grade B/C) pancreatic fistula between LDP and ODP groups. For all the other perioperative and postoperative outcomes, the metaanalysis confirmed that laparoscopic approach is comparable with the open one. The last meta-analysis was published in 2015 by Mehrabi et al. [25], with the aim of providing recommendation for clinical practice and research on the basis of surgical morbidity, such as pancreatic fistula, delayed gastric emptying and safety. Twenty-nine observational studies and 3,701 overall patients were included in the meta-analysis. Results showed superiority of LDP in terms of blood loss, time to first oral intake and hospital stay. All other parameters of operative morbidity and safety showed no difference. Data on oncologic radicality and effectiveness were limited. 
The retrospective nature of all published studies must be taken into account, because obviously it causes important biases, particularly in the selection of patients for laparoscopic or open approach. In fact, laparoscopic approach is frequently performed in case of benign or borderline lesions. Moreover, even in the case of LDP for malignancy, it is possible that patients candidate to this approach are well selected (less large tumor, absence on vascular infiltration, etc.) if compared with cases treated with open approach. Few retrospective studies focusing on LDP for pancreatic cancer have been published in the last years. Kooby et al. [26] performed a multicenter casecontrol study of 2,012 patients who underwent LDP and ODP for pancreatic cancer, with the aim to analyze perioperative and oncologic outcomes. The authors demonstrated that LDP and ODP had no significant differences in short- and long-term oncological outcomes, in terms of number of examined lymph nodes, margin-negative resection rate and overall survival. The authors concluded that LDP was at least equivalent to open resection from a complication and oncologic standpoint and, therefore, a potential approach in select patients with pancreatic cancer. Similar results were reported by Magge et al. [16] finding no difference in perioperative and oncological outcomes between ODP and LDP. Despite these results, however, there is insufficient evidence (absence of prospective RCTs) to support definitive conclusions on short- and long-term oncologic outcomes with regard to superiority of LDP over ODP. According to all these above-mentioned consideration, it seems clear that, in order to demonstrate the superiority of LDP compared to ODP, particularly in terms of oncological results, it is time to randomize. Two recent important national observational studies, published by French and Dutch groups $[27,28]$, confirmed this necessity; only a multicentric randomized control trial could provide the superiority of LDP, if compared with ODP.

Regarding the robotic approach for DP, some studies demonstrating its feasibility and safety have been published during the last years [29-33]. Moreover, in regards to eventual advantages of the use of robot for DP, 2 studies comparing LDP and ODP to robotic DP (RDP) have been published $[5,26]$. The first includes 20 RDPs and 25 LDPs for benign and borderline malignant lesions, with intent to preserve the spleen [5]; the authors reported a much higher splenic preservation rate with the robotic approach, even if the robotic approach was associated with longer postoperative times and about double the average cost per case compared to LDP. The other study by Daouadi et al. [29], including 30 RDP and 94 LDP pa- tients, was unable to demonstrate a significant reduction in blood loss, hospital stay or morbidity between the 2 groups. These data highlight some of the actual criticisms of the robotic approach - more cost without an obvious tangible advantage.

\section{Minimally Invasive PD}

Minimally invasive approach for PD today represents the most fascinating surgical challenge for pancreatic surgeons. Interest in laparoscopic PD (LPD) has continued to grow since its first description by Gagner and Pomp [34] in 1994, and larger series describing this technique limited to a few highly specialized centers have been described in the last few years [30, 35-43]. However, LPD is still not universally accepted, because the complexity of the procedure has questioned the safety and advantages over its open counterpart. Acceptance was probably slowed by both the inherent technical limitations of laparoscopy and the need for advanced laparoscopic skills, which were not always acquired during the training of pancreatic surgeons [44]. However, successful application of laparoscopy to other complex abdominal operations, availability of newer technologies, such as the da Vinci surgical system [45] and surgeon's motivation to pursue innovation and accept challenge [46] have all contributed to increase popularity and acceptance of minimally invasive PD in some centers. Boggi et al. [47] in a recent review underline this increasing popularity, showing that the number of LPD published between January 2012 and June 2013 exceeds that of LPD published in the previous 15 years.

In recent years, a large number of single-institution series of LPD have been performed [32-40]. All these studies have supported that LPD is feasible and safe in expert hands and in high-volume pancreatic centers. The real debate is if the minimally invasive approach can be considered a standard in performing PD and if it has the same outcomes of OPD. About this topic, 2 meta-analyses were published in 2014 [48, 49], in order to compare the intraoperative outcomes, postoperative complications, postoperative recovery and oncological safety in OPD and LPD. It is to be noted that no RCTs has been published, and the 2 meta-analyses consider only retrospective studies. The results of one of these meta-analysis [46] demonstrated that minimally invasive PD was associated with a reduction in estimated blood loss, wound infection and a shorter hospital stay, even if it was associated with a longer operative time; there were no signifi- 
cant differences in the likelihood of overall complications, pancreatic fistula, delayed gastric emptying, reoperation and mortality. Correa-Gallego et al. [48] recently published the second meta-analysis about this topic, including 6 retrospective studies and 542 patients (169 LPD and 373 OPD). Minimally invasive surgery was associated with a reduction in intraoperative blood loss, although longer operative times were observed; overall morbidity, reoperations and other specific complications were comparable, and hospital stay was significantly reduced in the LPD group. Moreover, $10 \%$ of patients in the LPD group required conversion to an open procedure; the majority of these were due to bleeding or the inability to obtain adequate vascular control laparoscopically. Regarding the results of these 2 meta-analysis, 2 considerations are necessary: (a) in absence of RCTs comparing open with minimally invasive $\mathrm{PD}$, there was an obvious selection bias: patients who underwent minimally invasive approach were more often affected by borderline tumors (mucinous cystic lesions, IPMN) or by more little tumor, if compared with patients selected for open PD, and (b) all the PDs with minimally invasive approach were performed in high-volume centers. In conclusion, LPD is currently a feasible option but at the moment it should be performed in high-volume pancreatic surgery centers and in selected patients (i.e., small cancer distant from major vessels or borderline lesions).

Considering the use of robot, it is common opinion between expert pancreatic and laparoscopic surgeons that in the case of PD, more than in the case of DP, the advent of robotics will give the impulse and the opportunity to improve the successful rate of minimally invasive approach. In fact, some technical problems routinely encountered during LPD requiring conversion to open surgery can be overcome with the use of robotic approach. For this reason, the recent literature clearly shows an increase of interest in the robotic approach for PD, more than the laparoscopic one. This phenomenon is evident looking at the literature: $90 \%$ of the minimally invasive DP literature focuses on conventional laparoscopy, with only $10 \%$ referencing as robotic approach, but if we compared this with the PubMed search results of the recent PD literature, we found that $29 \%$ of these featured robotic surgery [50]. However, it is important to note that these reports were all based on single-institutional experience, and evidence in the context of RCTs is not available. Zhang et al. [51] in 2013 published the first metaanalysis comparing robotic versus open PD; none of the included studies were randomized. This meta-analysis showed that overall complication rate was significantly

Minimally Invasive Pancreatic Resection: Is It Really the Future? lower in robotic group, as well as reoperation rate; there was no significant difference in postoperative pancreatic fistula incidence and mortality; the median conversion rate was $10 \%$. In conclusion, the results of meta-analysis favor the safety and efficacy of robotic PD in terms of clinical outcomes; however, more evidence of prospective, multicenter, RCTs is needed to further address the role of robotic technique in pancreatic surgery, not only for the safety reasons but also for the real overall benefits to patients if compared with open approach. Regarding the comparison between RPD and LPD, recently, a review by Orti-Rodriguez and Rahman [52] provides the first comparative review between LPD and robotic-PD (RPD). The authors found that the overall operating times were comparable in both the 2 groups. Surprisingly, the mean estimated blood loss was significantly higher in the robotic arms compared with the laparoscopic group. Similarly, the rates of enteric fistula (biliary, pancreatic and gastric) were also higher in the RPD group, even if the overall morbidity and mortality rates were comparable. Moreover, this review found that the conversion rate was significantly greater in the RPD group (11.56 vs. $5.63 \%)$. These results can be explained with the different expertise of the surgeons with the 2 different techniques: the skill level in years for the laparoscopic practitioners is far greater than that for relatively new robotic technology and is not comparable between the 2 groups. In addition to the different expertise, one of the major perceived drawbacks of robotic surgery is the associated cost, which is higher if compared with LPD (even if there are currently no studies that performed a direct cost-benefit comparison between the 2 surgical approaches).

As for minimally invasive DP, the real question is whether we consider this approach oncologically effective. Even in this case, no randomized studies are present in the literature, and so, current published series of LPD include patients with various diagnoses and embody a relative reluctance to use a laparoscopic approach in patients with pancreatic malignancy [33]. As such, there is a paucity of data on the oncologic outcomes of LPD and there are no comparative trials in the setting of pancreatic ductal adenocarcinoma. Buchs et al. [53] compared 33 patients undergoing RPD with 27 patients undergoing $\mathrm{OPD}$, demonstrating no difference in $\mathrm{R} 0$ resection and a higher number of nodes harvested in the minimally invasive group than in the OPD group. Chalikonda et al. [40] compared 14 patients undergoing RPD and 14 patients undergoing $\mathrm{OPD}$, demonstrating a lower proportion of patients with margin-positive resection in the robotic 
group than in the other one. In a more recent comparison, Asbun and Stauffer [37] included 39 patients undergoing LPD and 100 patients undergoing OPD for malignancy, showing no difference in R0 resection and a higher mean number of lymph nodes harvested in the laparoscopic group. Recently, a real first attempt to prove the oncological validity of minimally invasive approach has been done by Croome et al. [54]; the authors reviewed data for all patients undergoing LPD or OPD for pancreatic ductal adenocarcinoma at the Mayo Clinic between January 2008 and July 2013, with the aim to directly compare the oncological outcomes of the 2 procedures. Neoadjuvant therapy, tumor size, node positivity and margin-positive resection were similar in the 2 groups. However, there were a significantly higher proportion of patients in the OPD group who had a delay of greater than 90 days or did not receive adjuvant chemotherapy at all compared with that in the LPD group. Moreover, this study showed that even if there was not a significant difference in overall survival between the 2 groups, a significantly longer progression-free survival was seen in the LPD group than in the OPD group. The authors concluded that LPD is not only technically feasible and safe in the setting of adenocarcinoma but may also provide advantages such as shorter hospital stay and quick recovery, allowing patients to recover in a timeline manner and pursue adjuvant treatment options that are responsible of the longer progression free-survival observed in this subset of patients. However, in absence of RCTs, it is difficult to af- firm at the moment that LPD would surely offer the same or better oncological results if compared with open approach.

\section{Conclusions}

Considering all the available evidences in the literature, it is possible to affirm that minimally invasive approach for major pancreatic resections is feasible and safe in selected patients in expert centers: both robotic and laparoscopic approach can be used in expert hands for PD and DP, with no increased mortality and with some advantages in terms of perioperative outcomes and length of stay. However, due to the absence of prospective RCTs, it is impossible at the moment to affirm that minimally invasive approach is equal to open procedure or even the standard procedure for pancreatic surgical resections. In fact, many patients considered for laparoscopic approach are selected, with benign, borderline lesion or with small cancer without vascular involvement. Moreover, due to the paucity of patients affected by neoplastic disease treated with minimally invasive approach, data on long-term outcomes of tumor recurrence and patient survival are not well defined.

\section{Disclosure Statement}

The authors have no conflicts of interest to disclose.

\section{References}

1 Polychronidis A, Laftsidis P, Bounovas A, et al: Twenty years of laparoscopic cholecystectomy: Philippe Mouret - March 17, 1987. JSLS 2008;12:109-111.

2 Addeo P, Giulianotti PC: Update on laparoscopic pancreatectomy in 2010. Minerva Chir 2010;65:655-666.

3 Byrn JC, Schluender S, Divino CM, et al: Three-dimensional imaging improves surgical performance for both novice and experienced operators using the da Vinci robot system. Am J Surg 2007;193:519-522.

4 Boggi U, Signori S, De Lio N, et al: Feasibility of robotic pancreaticoduodenectomy. Br J Surg 2013;100:917-925.

5 Kang CM, Kim DH, Lee WJ, et al: Conventional laparoscopic and robot-assisted spleenpreserving pancreatectomy: does da Vinci have clinical advantages? Surg Endosc 2011; 25:2004-2009.

6 Cuschieri A, Jakimowicz JJ, van Spreeuwel J: Laparoscopic distal 70\% pancreatectomy and splenectomy for chronic pancreatitis. Ann Surg 1996;223:280-285.

7 Gagner M, Pomp A, Herrera MF: Early experience with laparoscopic resections of islet cell tumors. Surgery 1996;120:1051-1054.

8 Kooby DA, Gillespie T, Bentrem D, et al: Leftsided pancreatectomy: a multicenter comparison of laparoscopic and open approaches. Ann Surg 2008;248:438-446.

9 Røsok BI, Marangos IP, Kazaryan AM, et al: Single-centre experience of laparoscopic pancreatic surgery. Br J Surg 2010;97:902-909.

10 Vijan SS, Ahmed KA, Harmsen WS, et al: Laparoscopic vs open distal pancreatectomy: a single-institution comparative study. Arch Surg 2010;145:616-621.

11 Jayaraman S, Gonen M, Brennan MF, et al: Laparoscopic distal pancreatectomy: evolution of a technique at a single institution. J Am Coll Surg 2010;211:503-509.

12 Song KB, Kim SC, Park JB, et al: Single-center experience of laparoscopic left pancre- atic resection in 359 consecutive patients: changing the surgical paradigm of left pancreatic resection. Surg Endosc 2011;25: 3364-3372.

13 Baker MS, Bentrem DJ, Ujiki MB, et al: A prospective single institution comparison of perioperative outcomes for laparoscopic and open distal pancreatectomy. Surgery 2009; 146:635-643; discussion 643-645.

14 Finan KR, Cannon EE, Kim EJ, et al: Laparoscopic and open distal pancreatectomy: a comparison of outcomes. Am Surg 2009;75: 671-679; discussion 679-680.

15 DiNorcia J, Schrope BA, Lee MK, et al: Laparoscopic distal pancreatectomy offers shorter hospital stays with fewer complications. J Gastrointest Surg 2010;14:1804-1812.

$\checkmark 16$ Magge D, Gooding W, Choudry H, et al: Comparative effectiveness of minimally invasive and open distal pancreatectomy for ductal adenocarcinoma. JAMA Surg 2013;148: 525-531. 


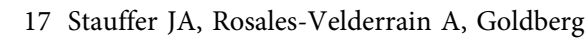
$\mathrm{RF}$, et al: Comparison of open with laparoscopic distal pancreatectomy: a single institution's transition over a 7-year period. HPB (Oxford) 2013;15:149-155.

18 Jusoh AC, Ammori BJ: Laparoscopic versus open distal pancreatectomy: a systematic review of comparative studies. Surg Endosc 2012;26:904-913.

19 Nigri GR, Rosman AS, Petrucciani N, et al: Meta-analysis of trials comparing minimally invasive and open distal pancreatectomies. Surg Endosc 2011;25:1642-1651.

20 Venkat R, Edil BH, Schulick RD, et al: Lapa- 32 roscopic distal pancreatectomy is associated with significantly less overall morbidity compared to the open technique: a systematic review and meta-analysis. Ann Surg 2012;255: 1048-1059.

21 Sui CJ, Li B, Yang JM, Wang SJ, et al: Laparoscopic versus open distal pancreatectomy: a meta-analysis. Asian J Surg 2012;35:1-8.

-22 Jin T, Altaf K, Xiong JJ, Huang W, et al: A systematic review and meta-analysis of studies comparing laparoscopic and open distal pancreatectomy. HPB (Oxford) 2012;14:711-724.

23 Xie K, Zhu YP, Xu XW, et al: Laparoscopic distal pancreatectomy is as safe and feasible as open procedure: a meta-analysis. World J Gastroenterol 2012;18:1959-1967.

-24 Pericleous S, Middleton N, McKay SC, et al: Systematic review and meta-analysis of casematched studies comparing open and laparoscopic distal pancreatectomy: is it a safe procedure? Pancreas 2012;41:993-1000.

25 Mehrabi A, Hafezi M, Arvin J, et al: A systematic review and meta-analysis of laparoscopic versus open distal pancreatectomy for benign and malignant lesions of the pancreas: it's time to randomize. Surgery 2015;157:45-55.

26 Kooby DA, Hawkins WG, Schmidt CM, et al: A multicenter analysis of distal pancreatectomy for adenocarcinoma: is laparoscopic resection appropriate? J Am Coll Surg 2010;210: 779-785, 786-787.

$\checkmark 27$ De Rooij T, Jilesen AP, Boerma D, et al: A nationwide comparison of laparoscopic and open distal pancreatectomy for benign and malignant disease. J Am Coll Surg 2015;220: 263-270.e1.

28 Sulpice L, Farges O, Goutte N, et al: Laparoscopic distal pancreatectomy for pancreatic ductal adenocarcinoma: time for a randomized controlled trial? Results of an all-inclusive national observational study. Ann Surg 2015;262:868-873; discussion 873-874.
29 Daouadi M, Zureikat AH, Zenati MS, et al: Robot-assisted minimally invasive distal pancreatectomy is superior to the laparoscopic technique. Ann Surg 2013;257:128-132.

30 Hwang HK, Kang CM, Chung YE, et al: Robot-assisted spleen-preserving distal pancreatectomy: a single surgeon's experiences and proposal of clinical application. Surg Endosc 2013;27:774-781.

-31 Suman P, Rutledge J, Yengpruksawan A: Robotic spleen preserving distal pancreatectomy is safe and feasible. Gastroenterology 2012; 142:S1060-S1061.

32 Waters JA, Canal DF, Wiebke EA, et al: Robotic distal pancreatectomy: cost effective? Surgery 2010;148:814-823.

33 Giulianotti PC, Sbrana F, Bianco FM, et al: Robot-assisted laparoscopic pancreatic surgery: single-surgeon experience. Surg Endosc 2010;24:1646-1657.

34 Gagner M, Pomp A: Laparoscopic pyloruspreserving pancreatoduodenectomy. Surg Endosc 1994;8:408-410.

35 Kendrick ML, Cusati D: Total laparoscopic pancreaticoduodenectomy: feasibility and outcome in an early experience. Arch Surg 2010;145:19-23.

36 Palanivelu C, Rajan PS, Rangarajan M, et al: Evolution in techniques of laparoscopic pancreaticoduodenectomy: a decade long experience from a tertiary center. J Hepatobiliary Pancreat Surg 2009;16:731-740.

-37 Asbun HJ, Stauffer JA: Laparoscopic vs open pancreaticoduodenectomy: overall outcomes and severity of complications using the accordion severity grading system. J Am Coll Surg 2012;215:810-819.

38 Zureikat AH, Moser AJ, Boone BA, et al: 250 robotic pancreatic resections: safety and feasibility. Ann Surg 2013;258:554-559; discussion 559-562.

39 Kim SC, Song KB, Jung YS, et al: Short-term clinical outcomes for 100 consecutive cases of laparoscopic pylorus-preserving pancreatoduodenectomy: improvement with surgical experience. Surg Endosc 2013;27:95-103.

40 Chalikonda S, Aguilar-Saavedra JR, Walsh RM: Laparoscopic robotic-assisted pancreaticoduodenectomy: a case-matched comparison with open resection. Surg Endosc 2012; 26:2397-2402.

41 Dulucq JL, Wintringer P, Mahajna A: Laparoscopic pancreaticoduodenectomy for benign and malignant diseases. Surg Endosc 2006;20: 1045-1050.
42 Zheng MH, Feng B, Lu AG, et al: Laparoscopic pancreaticoduodenectomy for ductal adenocarcinoma of common bile duct: a case report and literature review. Med Sci Monit 2006;12:CS57-CS60.

43 Pugliese R, Scandroglio I, Sansonna F, et al: Laparoscopic pancreaticoduodenectomy: a retrospective review of 19 cases. Surg Laparosc Endosc Percutan Tech 2008;18:13-18.

44 Subhas G, Mittal VK: Training minimal invasive approaches in hepatopancreatobilliary fellowship: the current status. HPB (Oxford) 2011;13:149-152.

45 Kim VB, Chapman WH, Albrecht RJ, et al: Early experience with telemanipulative robotassisted laparoscopic cholecystectomy using da Vinci. Surg Laparosc Endosc Percutan Tech 2002;12:33-40.

46 Fingerhut A: Surgical ego, the good, the bad, and the ugly. Surg Innov 2011;18:97-98.

47 Boggi U, Amorese G, Vistoli F, et al: Laparoscopic pancreaticoduodenectomy: a systematic literature review. Surg Endosc 2015;29: 9-23.

48 Correa-Gallego C, Dinkelspiel HE, Sulimanoff I, et al: Minimally-invasive vs open pancreaticoduodenectomy: systematic review and meta-analysis. J Am Coll Surg 2014;218: 129-139.

49 Qin H, Qiu J, Zhao Y, Pan G, Zeng Y: Does minimally-invasive pancreaticoduodenectomy have advantages over its open method? A meta-analysis of retrospective studies. PLoS One 2014;9:e104274.

50 Place TL, Nau P, Mezhir JJ: Minimally invasive pancreatectomy for cancer: a critical review of the current literature. J Gastrointest Surg 2015;19:375-386.

51 Zhang J, Wu WM, You L, Zhao YP: Robotic versus open pancreatectomy: a systematic review and meta-analysis. Ann Surg Oncol 2013;20:1774-1780.

52 Orti-Rodriguez RJ, Rahman SH: A comparative review between laparoscopic and robotic pancreaticoduodenectomies. Surg Laparosc Endosc Percutan Tech 2014;24:103-108.

53 Buchs NC, Addeo P, Bianco FM, et al: Robotic versus open pancreaticoduodenectomy: a comparative study at a single institution. World J Surg 2011;35:2739-2746.

54 Croome KP, Farnell MB, Que FG, et al: Total laparoscopic pancreaticoduodenectomy for pancreatic ductal adenocarcinoma: oncologic advantages over open approaches? Ann Surg 2014;260:633-638; discussion 638-640. 